\title{
Resurgence of one-point functions in a matrix model for 2D type IIA superstrings
}

\author{
Tsunehide Kuroki ${ }^{a, b}$ and Fumihiko Sugino ${ }^{c}$ \\ ${ }^{a}$ General Eduction, National Institute of Technology, Kagawa College, \\ 551 Kohda, Takuma-cho, Mitoyo, Kagawa 769-1192, Japan \\ ${ }^{b}$ Osaka City University Advanced Mathematical Institute (OCAMI), \\ 3-3-138 Sugimoto, Sumiyoshi-ku, Osaka 558-8585, Japan \\ ${ }^{c}$ Center for Theoretical Physics of the Universe, Institute for Basic Science (IBS), \\ Expo-ro 55, Yuseong-gu, Daejeon 34126, Republic of Korea \\ E-mail: t96ki@hotmail.com, fusugino@gmail.com
}

ABSTRACT: In the previous papers, the authors pointed out correspondence between a supersymmetric double-well matrix model and two-dimensional type IIA superstring theory on a Ramond-Ramond background. This was confirmed by agreement between planar correlation functions in the matrix model and tree-level amplitudes in the superstring theory. Furthermore, in the matrix model we computed one-point functions of singletrace operators to all orders of genus expansion in its double scaling limit, and found that the large-order behavior of this expansion is stringy and not Borel summable. In this paper, we discuss resurgence structure of these one-point functions and see cancellations of ambiguities in their trans-series. More precisely, we compute both series of ambiguities arising in a zero-instanton sector and in a one-instanton sector, and confirm how they cancel each other. In case that the original integration contour is a finite interval not passing through a saddle point, we have to choose an appropriate integration path in order for resurgence to work.

KEYwords: Matrix Models, Nonperturbative Effects, Supersymmetry Breaking, 2D Gravity

ARXIV EPRINT: 1901.10349 


\section{Contents}

1 Introduction 1

2 Review of the supersymmetric matrix model 3

2.1 Supersymmetry and large- $N$ limit 3

2.2 Correlation functions in fixed filling fraction 4

2.3 Correlation functions in fixed instanton sector 5

$\begin{array}{lll}3 & \text { One-point functions via Nicolai mapping } & 7\end{array}$

4 Ambiguities in the zero-instanton sector $\quad 10$

$\begin{array}{ll}4.1 \text { Genus expansion to all orders } & 10\end{array}$

4.2 Borel resummation 11

5 Ambiguities in the one-instanton sector $\quad 14$

$\begin{array}{lll}5.1 & \text { One-point functions in the one-instanton sector } & 14\end{array}$

$\begin{array}{ll}5.2 \text { Saddle point method } & 14\end{array}$

$\begin{array}{llr}6 & \text { Conclusions and discussions } & 18\end{array}$

\section{Introduction}

Resurgence [1-8] has attracted lots of attention by its intriguing property to make intimate connection between perturbative and nonperturbative quantities. From data of higher-order perturbative expansion, resurgence enables us to extract nonperturbative aspects. It has been investigated how resurgence works in each of various quantum theories, ${ }^{1}$ whereas we still do not know much about a unified picture or classification of their resurgence structure. $^{2}$ In addition, even in a specific model or theory, we have not clarified how resurgence structure changes depending on its physical observables. Namely, some observables may be strongly affected by nonperturbative effects and resurgence plays an important role in extracting nonperturbative information, while other observables may not and their perturbative series may behave too well to get insight into nonperturbative aspects by resurgent analysis.

Furthermore, we have not known much about relation between resurgence structure and physics. In general, nonperturbative dynamics is important to explain how interesting physical phenomena like quark confinement or symmetry breaking take place. When resurgence extracts some information on nonperturbative effects from perturbation theory, we

\footnotetext{
${ }^{1}$ Resurgence structure has been studied in various models and theories based on several motivations: see e.g. in quantum mechanics [9-35], string theories [36-52] as well as quantum field theories [53-89].

${ }^{2}$ Recent progress in this direction have been made, e.g. in [34].
} 
expect that some insights into such interesting physics are available. Thus, it is desirable to find various examples where resurgence helps us understand nonperturbative aspects of physics.

Based on these motivations, in this paper we study resurgence structure in a supersymmetric double-well matrix model with the action

$$
S=N \operatorname{tr}\left[\frac{1}{2} B^{2}+i B\left(\phi^{2}-\mu^{2}\right)+\bar{\psi}(\phi \psi+\psi \phi)\right],
$$

where $B$ and $\phi$ are $N \times N$ Hermitian matrices, and $\psi$ and $\bar{\psi}$ are $N \times N$ Grassmann-odd matrices. $\mu^{2}$ is a parameter of the model. The action $S$ is invariant under supersymmetry transformations generated by $Q$ and $\bar{Q}$ :

$$
\begin{aligned}
& Q \phi=\psi, \quad Q \psi=0, \quad Q \bar{\psi}=-i B, \quad Q B=0, \\
& \bar{Q} \phi=-\bar{\psi}, \quad \bar{Q} \bar{\psi}=0, \quad \bar{Q} \psi=-i B, \quad \bar{Q} B=0,
\end{aligned}
$$

which lead to the nilpotency: $Q^{2}=\bar{Q}^{2}=\{Q, \bar{Q}\}=0$. One of the most interesting features of this model is that the supersymmetry is spontaneously broken by nonperturbative effects in a certain large- $N$ limit called as double scaling limit (defined in (2.2)) [90, 91]. We also have proposed in [92] that this model under the double scaling limit gives nonperturbative formulation of type IIA superstring theory in two dimensions on a Ramond-Ramond background. From these, we can regard this model as an invaluable example of spontaneously broken target-space supersymmetry in string theory. In this paper, we concentrate on one-point functions of powers of matrix $\phi:\left\langle\frac{1}{N} \operatorname{tr} \phi^{n}\right\rangle$. In the previous work [93], it is shown that the operators with $n$ even $(n \in 2 \boldsymbol{N})$ are essentially supersymmetric, and $1 / N$ or genus expansions of their one-point functions are polynomials in the parameter $\mu^{2}$, terminating at some genus, which do not lead to any nonanalytic behavior in the double scaling limit. In [90], taking account of effects nonperturbative in $1 / N$, we have calculated the one-point function $\left\langle\frac{1}{N} \operatorname{tr}\left(\phi^{2}-\mu^{2}\right)\right\rangle$ (or equivalently $\left\langle\frac{1}{N} \operatorname{tr} B\right\rangle$ ) as an order parameter of the spontaneous supersymmetry breaking. On the other hand, the odd-power operators $(n \in 2 \boldsymbol{N}-1)$ are not supersymmetric, and genus expansions of their one-point functions exhibit stringy growth of the expansion coefficients as $(2 h)$ ! as genus $h$ grows. In this paper, we consider the one-point functions of the odd-power operators and study their resurgence structure. Since instantons in the matrix model (1.1) contribute to the order parameter $\left\langle\frac{1}{N} \operatorname{tr}\left(\phi^{2}-\mu^{2}\right)\right\rangle$ and trigger spontaneous supersymmetry breaking [90], our main interest is to clarify how resurgence structure for the odd-power operators is related to such nonperturbative physics.

Another advantage of considering our model (1.1) is that the existence of the Nicolai mapping [90]. Although resurgence requires data of large-order perturbation series, it is not easy to obtain such data in general. In case that a theory is supersymmetric, we may compute its perturbative expansion to all orders, but in turn it may be Borel summable and have trivial resurgence structure. One of nice approaches to overcome this issue is to introduce a parameter explicitly breaking supersymmetry [27-31]. In [96] and this paper, we propose another way to obtain perturbative expansion to all orders for 
resurgence: we consider nonsupersymmetric quantities in a supersymmetric model. In fact, even in calculation of nonsupersymmetric quantities in (1.1), the Nicolai mapping is available reflecting the existence of supersymmetry in the action. This kind of idea will be useful, in particular, in supersymmetric field theories as in [82-88].

The organization of this paper is as follows. In the next section, we give a brief review of the supersymmetric double-well matrix model. Correlation functions are expressed in terms of eigenvalues of the matrix $\phi$ and are defined in each instanton sector. In section 3 , we explain how to compute the one-point functions of odd powers of $\phi$ by utilizing the Nicolai mapping. In section 4 , we consider contribution from the zero-instanton sector to the one-point functions, and find that there exists a series of ambiguities after applying the Borel resummation technique. Then, in section 5 we see that contribution from the one-instanton sector also has another series of ambiguities, and confirm that these series exactly cancel each other at the leading and next-to-leading orders. The last section is devoted to conclusions and discussions.

\section{Review of the supersymmetric matrix model}

In this section, we give a brief review of the supersymmetric double-well matrix model defined by the action (1.1), which has been proposed as a nonperturbative formulation of type IIA superstring theory in two dimensions.

\subsection{Supersymmetry and large- $N$ limit}

After integrating out the auxiliary variable $B$ in (1.1), the scalar potential of $\phi$ reads

$$
V(\phi)=\frac{1}{2}\left(\phi^{2}-\mu^{2}\right)^{2} .
$$

In the planar limit $\left(N \rightarrow \infty\right.$ with $\mu^{2}$ fixed) of the matrix model, there are infinitely degenerate supersymmetric vacua parametrized by filling fractions $\left(\nu_{+}, \nu_{-}\right)$for $\mu^{2} \geq 2$. The filling fractions represent configurations that $\nu_{ \pm} N$ of the eigenvalues of $\phi$ are around the minimum $\pm|\mu|$ of the double-well potential (2.1) [94, 95]. On the other hand, for $\mu^{2}<2$ we have a unique vacuum which breaks the supersymmetry. The boundary $\mu^{2}=2$ is a critical point at which the third-order phase transition occurs. In the planar limit, it is explicitly seen in $[92,93]$ that the result of several types of correlation functions in the matrix model reproduces the tree amplitudes in two-dimensional type IIA superstring theory on a nontrivial Ramond-Ramond background. In addition, we have considered the following double scaling limit [90] that approaches the critical point from the inside of the supersymmetric phase:

$$
N \rightarrow \infty, \quad \mu^{2} \rightarrow 2+0, \quad \text { with } \quad s=N^{\frac{2}{3}}\left(\mu^{2}-2\right): \text { fixed }
$$

This limit of the matrix model is expected to provide a nonperturbative formulation of the superstring theory with string coupling constant $g_{s}$ proportional to $s^{-\frac{3}{2}}$. From this viewpoint the planar limit mentioned above is regarded as $g_{s} \rightarrow 0$ limit. In fact, in [96] one-point functions for the single-trace operators of powers of $\phi$ are explicitly calculated at 
arbitrary genus and found to be finite at each genus under the double scaling limit (2.2). In $[90,91]$, contribution from matrix-model instantons (isolated eigenvalues of $\phi$ located around the top of the effective potential) to the free energy is found to be also finite and to have a factor $\exp \left(-C / g_{s}\right)$ with a constant $C$ of $\mathcal{O}(1)$. This form is typical of solitonic objects in string theory (D-branes). The correspondence between isolated eigenvalues and solitons is also observed in well-established bosonic noncritical string theories [101-108]. The most interesting feature of the model ever found is that these instantons cause spontaneous supersymmetry breaking in the matrix model, which implies violation of target-space supersymmetry induced by nonperturbative effects in the corresponding superstring theory. The aim of this paper is to investigate connection between contribution from higher genus in the absence of the instanton (in the zero-instanton sector) and that from the one-instanton sector through the one-point functions.

\subsection{Correlation functions in fixed filling fraction}

In this subsection, we define correlation functions of our model (1.1) in a fixed filling fraction. First, the partition function is expressed in terms of the eigenvalues of $\phi$ as follows [90, 93]:

$$
\begin{aligned}
Z & \equiv(-1)^{N^{2}} \int d^{N^{2}} B d^{N^{2}} \phi\left(d^{N^{2}} \psi d^{N^{2}} \bar{\psi}\right) e^{-S} \\
& =\tilde{C}_{N} \int_{-\infty}^{\infty}\left(\prod_{i=1}^{N} 2 \lambda_{i} d \lambda_{i}\right) \triangle\left(\lambda^{2}\right)^{2} e^{-N \sum_{i=1}^{N} \frac{1}{2}\left(\lambda_{i}^{2}-\mu^{2}\right)^{2}}
\end{aligned}
$$

where the normalization of the integration measure is fixed as

$$
\begin{aligned}
\int d^{N^{2}} \phi e^{-N \operatorname{tr}\left(\frac{1}{2} \phi^{2}\right)}=\int d^{N^{2}} B e^{-N \operatorname{tr}\left(\frac{1}{2} B^{2}\right)} & =1, \\
(-1)^{N^{2}} \int\left(d^{N^{2}} \psi d^{N^{2}} \bar{\psi}\right) e^{-N \operatorname{tr}(\bar{\psi} \psi)} & =1 .
\end{aligned}
$$

$\tilde{C}_{N}$ is a constant dependent only on $N: \tilde{C}_{N}=(2 \pi)^{-\frac{N}{2}} N^{\frac{N^{2}}{2}}\left(\prod_{k=0}^{N} k !\right)^{-1}[95]$, and $\triangle(x)$ stands for the Vandermonde determinant for eigenvalues $x_{i}(i=1, \cdots, N): \triangle(x) \equiv$ $\prod_{i>j}\left(x_{i}-x_{j}\right)$. By dividing the integration region of each $\lambda_{i}$ according to the filling fraction, the total partition function can be expressed as a sum of each partition function with a fixed filling fraction:

$$
\begin{aligned}
Z & =\sum_{\nu_{-} N=0}^{N} \frac{N !}{\left(\nu_{+} N\right) !\left(\nu_{-} N\right) !} Z_{\left(\nu_{+}, \nu_{-}\right)}, \\
Z_{\left(\nu_{+}, \nu_{-}\right)} & \equiv \tilde{C}_{N} \int_{0}^{\infty}\left(\prod_{i=1}^{\nu_{+} N} 2 \lambda_{i} d \lambda_{i}\right) \int_{-\infty}^{0}\left(\prod_{j=\nu_{+} N+1}^{N} 2 \lambda_{j} d \lambda_{j}\right) \triangle\left(\lambda^{2}\right)^{2} e^{-N \sum_{m=1}^{N} \frac{1}{2}\left(\lambda_{m}^{2}-\mu^{2}\right)^{2}} .
\end{aligned}
$$

By changing the integration variables $\lambda_{j} \rightarrow-\lambda_{j}\left(j=\nu_{+} N+1, \cdots, N\right)$, it is easy to find that $Z_{\left(\nu_{+}, \nu_{-}\right)}=(-1)^{\nu_{-} N} Z_{(1,0)}$ and the total partition function vanishes. 
Next, we define the correlation function of $K$ single-trace operators $\frac{1}{N} \operatorname{tr} \mathcal{O}_{a}(\phi)(a=$ $1, \cdots, K)$ in the filling fraction $\left(\nu_{+}, \nu_{-}\right)$as

$$
\begin{aligned}
\left\langle\prod_{a=1}^{K} \frac{1}{N} \operatorname{tr} \mathcal{O}_{a}(\phi)\right\rangle^{\left(\nu_{+}, \nu_{-}\right)} \equiv & \frac{\tilde{C}_{N}}{Z_{\left(\nu_{+}, \nu_{-}\right)}} \int_{0}^{\infty}\left(\prod_{i=1}^{\nu_{+} N} 2 \lambda_{i} d \lambda_{i}\right) \int_{-\infty}^{0}\left(\prod_{j=\nu_{+}+N^{\prime}}^{N} 2 \lambda_{j} d \lambda_{j}\right) \triangle\left(\lambda^{2}\right)^{2} \\
& \times\left(\prod_{a=1}^{K} \frac{1}{N} \sum_{i=1}^{N} \mathcal{O}_{a}\left(\lambda_{i}\right)\right) e^{-N \sum_{m=1}^{N} \frac{1}{2}\left(\lambda_{m}^{2}-\mu^{2}\right)^{2}}
\end{aligned}
$$

and express its connected part in the $1 / N$-expansion:

$$
\left\langle\prod_{a=1}^{K} \frac{1}{N} \operatorname{tr} \mathcal{O}_{a}(\phi)\right\rangle_{C}^{\left(\nu_{+}, \nu_{-}\right)}=\sum_{h=0}^{\infty} \frac{1}{N^{2 h+2 K-2}}\left\langle\prod_{a=1}^{K} \frac{1}{N} \operatorname{tr} \mathcal{O}_{a}(\phi)\right\rangle_{C, h}^{\left(\nu_{+}, \nu_{-}\right)} .
$$

$\langle\cdot\rangle_{C, h}^{\left(\nu_{+}, \nu_{-}\right)}$denotes the connected correlation function on a handle- $h$ random surface with the $N$-dependence factored out; i.e., the quantity of $\mathcal{O}\left(N^{0}\right)$. Let us consider the case where $\mathcal{O}_{a}(\phi)$ are polynomials of $\phi$. Operators $\frac{1}{N} \operatorname{tr} B^{k}$ or (linear combinations of) $\frac{1}{N} \operatorname{tr} \phi^{2 k}$ $(k \in N \cup\{0\})$ are invariant under the supersymmetries (1.2). For these operators, multipoint functions at the planar level $(h=0)$ and higher-genus one-point functions do not exhibit any nonanalytic behavior as $s \rightarrow 0$ [93, 96], which is characteristic of protection by supersymmetry. On the other hand, operators of odd powers: $\frac{1}{N} \operatorname{tr} \phi^{2 k+1}(k \in N \cup\{0\})$ are not invariant under either of $Q$ or $\bar{Q}$, and their correlation functions have nontrivial dependence on $s$ [96] as we will mention in the next section. For simplicity, in the following we focus on the one-point function of the odd-power operators: $(2.7)$ with $K=1, \mathcal{O}_{1}(\phi)=$ $\phi^{2 k+1}$ in the filling fraction ${ }^{3}\left(\nu_{+}, \nu_{-}\right)=(1,0)$.

\subsection{Correlation functions in fixed instanton sector}

In this subsection, we divide correlation functions in the $(1,0)$ sector into contributions from definite instanton numbers as done in [90]. In (2.5), the partition function $Z_{(1,0)}$ with the filling fraction $(1,0)$ is expressed as the integrations of $N$ eigenvalues along the positive real axis. The eigenvalue distribution in the planar limit is given as [93, 94]

$$
\left\langle\left.\left.\frac{1}{N} \sum_{i=1}^{N} \delta\left(x-\lambda_{i}\right)\right|^{(1,0)}\right|_{\text {planar }}= \begin{cases}\frac{x}{\pi} \sqrt{\left(x^{2}-a^{2}\right)\left(b^{2}-x^{2}\right)} & (a<x<b) \\ 0 & \text { (otherwise) }\end{cases}\right.
$$

with $a=\sqrt{\mu^{2}-2}$ and $b=\sqrt{\mu^{2}+2}$, which means that all the eigenvalues are confined in the interval $[a, b]$. Dividing the integration region of each eigenvalue $\boldsymbol{R}_{+}=[0, \infty)$ into the inside and outside of the support:

$$
\int_{0}^{\infty} d \lambda_{i}=\int_{a}^{b} d \lambda_{i}+\int_{\boldsymbol{R}_{+} \backslash[a, b]} d \lambda_{i}
$$

\footnotetext{
${ }^{3}$ It is shown in [93] that at least at the planar level $(h=0)$ and up to the three-point functions $(1 \leq K \leq$ $3)$, it is easy to recover filling fraction dependence of correlation functions from those in $\left(\nu_{+}, \nu_{-}\right)=(1,0)$.
} 
we decompose the partition function as

$$
\begin{aligned}
Z_{(1,0)} & =\left.\sum_{p=0}^{N} Z_{(1,0)}\right|_{p \text {-inst. }}, \\
\left.Z_{(1,0)}\right|_{p \text {-inst. }} & =\left(\begin{array}{c}
N \\
p
\end{array}\right) \tilde{C}_{N} \int_{a}^{b} \prod_{i=1}^{N-p} 2 \lambda_{i} d \lambda_{i} \int_{\boldsymbol{R}_{+} \backslash[a, b]} \prod_{i=1}^{p} 2 \lambda_{j} d \lambda_{j} \Delta\left(\lambda^{2}\right)^{2} e^{-N \sum_{i=1}^{N} \frac{1}{2}\left(\lambda_{i}^{2}-\mu^{2}\right)^{2}} .
\end{aligned}
$$

Each contribution with fixed $p$ is regarded as the partition function in the $p$-instanton sector. In fact, an instanton in our model corresponds to a saddle point of effective potential $V_{\text {eff }}\left(\lambda_{i}\right)$ with respect to a single eigenvalue $\lambda_{i}$, which is obtained by integrating out all the eigenvalues other than $\lambda_{i}$ in (2.3). Its saddle point turns out to be the origin $\lambda_{i}=0$ [90]. For large $s$ (small $g_{s}$ ) under the double scaling limit (2.2), the main contribution from the outside of the support $\boldsymbol{R}_{+} \backslash[a, b]$ is provided by such an instanton located at the origin. Then as mentioned in Introduction, leading contribution from the instanton takes the form of $\exp \left(-C / g_{s}\right)$. Correlation functions in the $p$-instanton sector can also be defined in a similar manner:

$$
\langle\mathcal{O}\rangle^{(1,0)}=\left.\sum_{p=0}^{N} \frac{\left.Z_{(1,0)}\right|_{p \text {-inst. }}}{Z_{(1,0)}}\langle\langle\mathcal{O}\rangle\rangle^{(1,0)}\right|_{p \text {-inst. }},
$$

where $\left.\langle\langle\mathcal{O}\rangle\rangle^{(1,0)}\right|_{p \text {-inst. }}$ denotes an expectation value of $\mathcal{O}$ within the $p$-instanton configurations normalized by $\left.Z_{(1,0)}\right|_{p \text {-inst. }}$. According to [90, 91], the partition functions behave as

$$
\left.Z_{(1,0)}\right|_{0 \text {-inst. }}=1,\left.\quad Z_{(1,0)}\right|_{p \text {-inst. }}=\left(\frac{e^{-\frac{4}{3} s^{\frac{3}{2}}}}{16 \pi s^{\frac{3}{2}}}\right)^{p} \times\left[1+\mathcal{O}\left(s^{-\frac{3}{2}}\right)\right]
$$

in the double scaling limit with $s$ finite but large, whereas $\left.\langle\langle\mathcal{O}\rangle\rangle^{(1,0)}\right|_{p \text {-inst. }}$ has no such exponential suppression. Hence (2.11) is a trans-series expanded by the instanton weight $e^{-\frac{4}{3} s^{\frac{3}{2}}} /\left(16 \pi s^{\frac{3}{2}}\right)$ :

$$
\begin{aligned}
\langle\mathcal{O}\rangle^{(1,0)}= & \left.\left.\left.\langle\langle\mathcal{O}\rangle\rangle^{(1,0)}\right|_{0 \text {-inst. }}(\langle\mathcal{O}\rangle\rangle^{(1,0)}\right|_{1 \text {-inst. }}-\left.\langle\langle\mathcal{O}\rangle\rangle^{(1,0)}\right|_{0 \text {-inst. }}\right) \\
& \left.+\left.Z_{(1,0)}\right|_{1 \text {-inst. }}\right) \\
& +\left.Z_{(1,0)}\right|_{2 \text {-inst. }}\left(\left.\langle\langle\mathcal{O}\rangle\rangle^{(1,0)}\right|_{2 \text {-inst. }}-\left.\langle\langle\mathcal{O}\rangle\rangle^{(1,0)}\right|_{0 \text {-inst. }}\right) \\
& +\left(\left.Z_{(1,0)}\right|_{1 \text {-inst. }}\right)^{2}\left(-\left.\langle\langle\mathcal{O}\rangle\rangle^{(1,0)}\right|_{1 \text {-inst. }}+\left.\langle\langle\mathcal{O}\rangle\rangle^{(1,0)}\right|_{0 \text {-inst. }}\right) \\
& +(\text { contribution from the total instanton number } p \geq 3),
\end{aligned}
$$

where the first line on the r.h.s. has no exponential suppression, while other lines have according to (2.12). In the previous work [96], we have computed the one-point function $\left.\langle\langle\mathcal{O}\rangle\rangle^{(1,0)}\right|_{0 \text {-inst. }}$ to all orders in expansion by $g_{s}^{2} \propto s^{-3}$ for $\mathcal{O}=\operatorname{tr} \phi^{n}$. In what follows we will 
find that for odd $n$, the Borel resummation of this expansion has a series of ambiguities, but explicitly show that it is indeed canceled by another series of ambiguities arising in the one-instanton sector given in the second line in (2.13) up to the next-to-leading order. This provides a strong support that there is no ambiguity in the trans-series form up to the instanton number one.

In the following, we use the notation:

$$
\begin{aligned}
& \left.\langle\mathcal{O}\rangle^{(1,0)}\right|_{0 \text {-inst. }}=\left.\langle\langle\mathcal{O}\rangle\rangle^{(1,0)}\right|_{0 \text {-inst. }}, \\
& \left.\langle\mathcal{O}\rangle^{(1,0)}\right|_{1 \text {-inst. }}=\left.Z_{(1,0)}\right|_{1 \text {-inst. }}\left(\left.\langle\langle\mathcal{O}\rangle\rangle^{(1,0)}\right|_{1 \text {-inst. }}-\left.\langle\langle\mathcal{O}\rangle\rangle^{(1,0)}\right|_{0 \text {-inst. }}\right) .
\end{aligned}
$$

\section{One-point functions via Nicolai mapping}

In this section, following the derivation in [96], we express the one-point function $\left\langle\frac{1}{N} \operatorname{tr} \phi^{2 k+1}\right\rangle^{(1,0)}$ in terms of quantities in the Gaussian matrix model. Let us first consider the $\phi^{2}$-resolvent

$$
\left\langle R_{2}\left(z^{2}\right)\right\rangle^{(1,0)}=\left\langle\frac{1}{N} \operatorname{tr} \frac{1}{z^{2}-\phi^{2}}\right\rangle^{(1,0)}
$$

In terms of the eigenvalues, $R_{2}\left(z^{2}\right)$ becomes

$$
\frac{1}{N} \sum_{i=1}^{N} \frac{1}{z^{2}-\lambda_{i}^{2}}=\frac{1}{N} \frac{1}{2 z} \sum_{i=1}^{N}\left(\frac{1}{z-\lambda_{i}}+\frac{1}{z+\lambda_{i}}\right),
$$

and $1 /\left(z-\lambda_{i}\right)\left(1 /\left(z+\lambda_{i}\right)\right)$ has poles only on the positive (negative) real axis for the filling fraction $(1,0)$. This leads to

$$
\left\langle\frac{1}{N} \operatorname{tr} \phi^{2 k+1}\right\rangle^{(1,0)}=\oint_{C_{0}} \frac{d z}{2 \pi i} 2 z^{2 k+2}\left\langle R_{2}\left(z^{2}\right)\right\rangle^{(1,0)},
$$

where $C_{0}$ is a contour which encloses only the poles at $z=\lambda_{i}$ for ${ }^{\forall} i$ counterclockwise. In particular, in the zero-instanton sector, $\lambda_{i}$ 's are all confined in the interval $[a, b]$, and therefore,

$$
\left.\left\langle\frac{1}{N} \operatorname{tr} \phi^{2 k+1}\right\rangle^{(1,0)}\right|_{0-\text { inst. }}=\oint_{C} \frac{d z}{2 \pi i} 2 z^{2 k+2}\left\langle R_{2}\left(z^{2}\right)\right\rangle^{(1,0)},
$$

where $C$ denotes a contour encircling the interval $[a, b]$ counterclockwise as depicted in figure 1. $C$ does not contain $z=0$ inside, and hence contribution from the instanton at the origin is not included in (3.4). Note that the $\phi^{2}$-resolvent is mapped to the resolvent in the Gaussian matrix model. In fact, the Nicolai mapping $x_{i}=\mu^{2}-\lambda_{i}^{2}(i=1, \cdots, N)$ recasts the partition function $Z_{(1,0)}$ and the one-point function (2.6) with $K=1$ in the filling fraction $(1,0)$ as

$$
Z_{(1,0)}=\tilde{C}_{N} \int_{-\infty}^{\mu^{2}}\left(\prod_{i=1}^{N} d x_{i}\right) \triangle(x)^{2} e^{-N \sum_{i=1}^{N} \frac{1}{2} x_{i}^{2}} \equiv Z^{\left(\mathrm{G}^{\prime}\right)}
$$




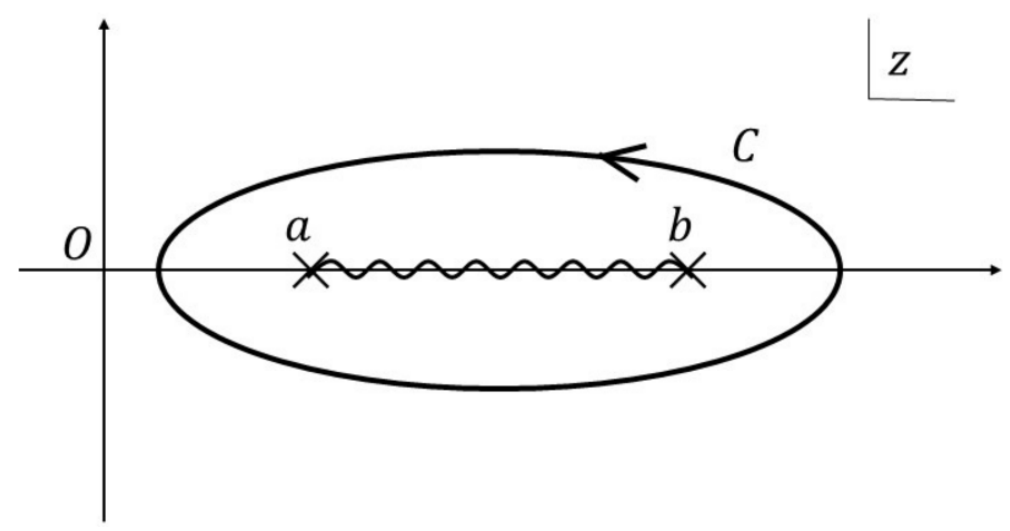

Figure 1. Integration contour $C$ on the complex $z$-plane.

and

$$
\left\langle\frac{1}{N} \operatorname{tr} \phi^{2 k+1}\right\rangle^{(1,0)}=\frac{\tilde{C}_{N}}{Z^{\left(\mathrm{G}^{\prime}\right)}} \int_{-\infty}^{\mu^{2}}\left(\prod_{i=1}^{N} d x_{i}\right) \triangle(x)^{2}\left(\frac{1}{N} \sum_{i=1}^{N}\left(\mu^{2}-x_{i}\right)^{k+\frac{1}{2}}\right) e^{-N \sum_{i=1}^{N} \frac{1}{2} x_{i}^{2}},
$$

respectively. Differently from the standard Gaussian matrix model, the integrals of the eigenvalues $x_{i}$ are not over the entire real axis, but are bounded from the above by $\mu^{2}$. The superscript (G') indicates a quantity in such a Gaussian matrix model. By introducing an $N \times N$ Hermitian matrix $M$ whose eigenvalues are $x_{i}(i=1, \cdots, N),(3.6)$ can be written as

$$
\left\langle\frac{1}{N} \operatorname{tr} \phi^{2 k+1}\right\rangle^{(1,0)}=-\oint_{C_{0}} \frac{d z}{2 \pi i} 2 z^{2 k+2}\left\langle R_{M}\left(\mu^{2}-z^{2}\right)\right\rangle^{\left(\mathrm{G}^{\prime}\right)}
$$

and

$$
\left.\left\langle\frac{1}{N} \operatorname{tr} \phi^{2 k+1}\right\rangle^{(1,0)}\right|_{0-\text { inst. }}=-\oint_{C} \frac{d z}{2 \pi i} 2 z^{2 k+2}\left\langle R_{M}\left(\mu^{2}-z^{2}\right)\right\rangle^{\left(\mathrm{G}^{\prime}\right)}
$$

where $R_{M}(x) \equiv \frac{1}{N} \operatorname{tr} \frac{1}{x-M}$ and the expectation value $\langle\cdot\rangle^{\left(\mathrm{G}^{\prime}\right)}$ is taken in the Gaussian matrix model (3.5).

It is also useful to express the one-point function by introducing the orthogonal polynomials $P_{n}(x)(n=0,1, \cdots)$ associated with the Gaussian matrix model (3.5) [90]:

$$
P_{n}(x)=x^{n}+\sum_{i=1}^{n-1} p_{n}^{(i)} x^{i}
$$

with $p_{n}^{(i)}$ coefficients, which satisfies the orthogonality

$$
\int_{-\infty}^{\mu^{2}} d x e^{-\frac{N}{2} x^{2}} P_{m}(x) P_{n}(x)=h_{m} \delta_{m n}
$$


and the recursion relation

$$
x P_{n}(x)=P_{n+1}(x)+S_{n} P_{n}(x)+R_{n} P_{n-1}(x) .
$$

Then (3.5) and (3.6) are expressed as

$$
Z_{(1,0)}=\tilde{C}_{N} N ! \prod_{n=0}^{N-1} h_{n}
$$

and

$$
\left\langle\frac{1}{N} \operatorname{tr} \phi^{2 k+1}\right\rangle^{(1,0)}=\frac{1}{N} \sum_{n=0}^{N-1} \frac{1}{h_{n}} \int_{-\infty}^{\mu^{2}} d x\left(\mu^{2}-x\right)^{k+\frac{1}{2}} P_{n}(x)^{2} e^{-\frac{N}{2} x^{2}} .
$$

Likewise eigenvalue distribution of the Gaussian matrix model

$$
\rho_{M}^{\left(\mathrm{G}^{\prime}\right)}(x) \equiv\left\langle\frac{1}{N} \operatorname{tr} \delta(x-M)\right\rangle^{\left(\mathrm{G}^{\prime}\right)}=\left\langle\frac{1}{N} \sum_{i=1}^{N} \delta\left(x-x_{i}\right)\right\rangle^{\left(\mathrm{G}^{\prime}\right)}
$$

becomes

$$
\rho_{M}^{\left(\mathrm{G}^{\prime}\right)}(x)=\frac{1}{N} \sum_{n=0}^{N-1} \frac{1}{h_{n}} P_{n}(x)^{2} e^{-\frac{N}{2} x^{2}} .
$$

From (3.13) and (3.15), we find a formula of the one-point function as an integral of the eigenvalue distribution

$$
\left\langle\frac{1}{N} \operatorname{tr} \phi^{2 k+1}\right\rangle^{(1,0)}=\int_{-\infty}^{\mu^{2}} d x\left(\mu^{2}-x\right)^{k+\frac{1}{2}} \rho_{M}^{\left(\mathrm{G}^{\prime}\right)}(x) .
$$

In [90], the orthogonal polynomials $P_{n}(x)$ are expressed in terms of the orthogonal polynomials $P_{n}^{(\mathrm{H})}(x)$ in the standard Gaussian matrix model (without the upper bound for eigenvalues). $P_{n}^{(\mathrm{H})}(x)$ is also a monic polynomial of degree $n$ satisfying

$$
\begin{aligned}
\int_{-\infty}^{\infty} d x e^{-\frac{N}{2} x^{2}} P_{m}^{(\mathrm{H})}(x) P_{n}^{(\mathrm{H})}(x) & =h_{n}^{(\mathrm{H})} \delta_{m n}, \\
x P_{n}^{(\mathrm{H})}(x) & =P_{n+1}^{(\mathrm{H})}(x)+S_{n}^{(\mathrm{H})} P_{n}^{(\mathrm{H})}(x)+R_{n}^{(\mathrm{H})} P_{n-1}^{(\mathrm{H})}(x) \quad\left(S_{n}^{(\mathrm{H})}=0\right) .
\end{aligned}
$$

We determine differences $\tilde{P}_{n}(x)=P_{n}(x)-P_{n}^{(\mathrm{H})}(x), \tilde{h}_{n}=h_{n}-h_{n}^{(\mathrm{H})}, \tilde{S}_{n}=S_{n}-S_{n}^{(\mathrm{H})}=S_{n}$, $\tilde{R}_{n}=R_{n}-R_{n}^{(\mathrm{H})}$ by taking account of the boundary effect of (3.10) in an iterative manner:

$$
\begin{aligned}
\tilde{P}_{n}(x) & =\tilde{P}_{n}^{(1)}(x)+\tilde{P}_{n}^{(2)}(x)+\cdots, \\
\tilde{S}_{n} & =\tilde{S}_{n}^{(1)}+\tilde{S}_{n}^{(2)}+\cdots, \\
\tilde{R}_{n} & =\tilde{R}_{n}^{(1)}+\tilde{R}_{n}^{(2)}+\cdots, \\
\tilde{h}_{n} & =\tilde{h}_{n}^{(1)}+\tilde{h}_{n}^{(2)}+\cdots,
\end{aligned}
$$


where the numbers $(1),(2), \cdots$ denote the steps of the iteration and turn out to correspond to the instanton numbers of the contributions [90]. Namely, quantities with the number $(p)$ are suppressed by the factor $\exp \left(-4 p s^{3 / 2} / 3\right)$ as $s$ grows as in (2.12).

Applying this expansion to the eigenvalue distribution (3.15) with

$$
\tilde{L}_{n}(x) \equiv \frac{\tilde{P}_{n}(x)}{P_{n}^{(\mathrm{H})}(x)}=\tilde{L}_{n}^{(1)}(x)+\tilde{L}_{n}^{(2)}(x)+\cdots,
$$

we obtain

$$
\begin{aligned}
\tilde{\rho}_{M}(x) & \equiv \rho_{M}^{\left(\mathrm{G}^{\prime}\right)}(x)-\rho_{M}^{(\mathrm{G})}(x) \\
& =\frac{1}{N} \sum_{n=0}^{N-1} \frac{1}{h_{n}^{(\mathrm{H})}} P_{n}^{(\mathrm{H})}(x)^{2} e^{-\frac{N}{2} x^{2}}\left(2 \tilde{L}_{n}^{(1)}(x)-\frac{\tilde{h}_{n}^{(1)}}{h_{n}^{(\mathrm{H})}}+\cdots\right),
\end{aligned}
$$

where $\rho_{M}^{(\mathrm{G})}(x)$ is the eigenvalue distribution of the standard Gaussian matrix model, and the ellipsis represents contribution from higher instanton numbers $(p \geq 2)$ [90]. Then the one-point function (3.16) is decomposed as

$$
\left\langle\frac{1}{N} \operatorname{tr} \phi^{2 k+1}\right\rangle^{(1,0)}=\int_{-\infty}^{\mu^{2}} d x\left(\mu^{2}-x\right)^{k+\frac{1}{2}} \rho_{M}^{(\mathrm{G})}(x)+\int_{-\infty}^{\mu^{2}} d x\left(\mu^{2}-x\right)^{k+\frac{1}{2}} \tilde{\rho}_{M}(x) .
$$

From straightforward calculation similar to what is done in section 5 in [90], the second term of the r.h.s. turns out to be a quantity with higher instanton numbers $(p \geq 2)$, and can be neglected as far as cancellation between the zero- and one-instanton sectors is concerned:

$$
\left.\left\langle\frac{1}{N} \operatorname{tr} \phi^{2 k+1}\right\rangle^{(1,0)}\right|_{0 \text {-inst. }+1 \text {-inst. }}=\int_{-\infty}^{\mu^{2}} d x\left(\mu^{2}-x\right)^{k+\frac{1}{2}} \rho_{M}^{(\mathrm{G})}(x) .
$$

By the same reason, we can replace the resolvent in (3.8) by that of the standard Gaussian matrix model (with the superscript $(G)$ ):

$$
\left.\left\langle\frac{1}{N} \operatorname{tr} \phi^{2 k+1}\right\rangle^{(1,0)}\right|_{0 \text {-inst. }}=-\oint_{C} \frac{d z}{2 \pi i} 2 z^{2 k+2}\left\langle R_{M}\left(\mu^{2}-z^{2}\right)\right\rangle^{(\mathrm{G})} .
$$

\section{Ambiguities in the zero-instanton sector}

In [96], the all-order result of genus expansion of the one-point functions $\left\langle\frac{1}{N} \operatorname{tr} \phi^{2 k+1}\right\rangle^{(1,0)}$ is obtained at the zero-instanton sector in the double scaling limit (2.2). In this section, we apply the Borel resummation technique to the result and find that ambiguities arise.

\subsection{Genus expansion to all orders}

For the resolvent of the standard Gaussian matrix model $\left\langle R_{M}(z)\right\rangle^{(\mathrm{G})}$, the expression of genus expansion is obtained at arbitrary genus in the literature e.g. [97]. In [96], utilizing 
the result there to (3.23), we have arrived at the expression

$$
\begin{aligned}
& \left\langle\frac{1}{N} \operatorname{tr} \phi^{2 k+1}\right\rangle^{(1,0)} \mid \\
& =N^{-\frac{2}{3}(k+2)} \frac{\Gamma\left(k+\frac{3}{2}\right)}{2 \pi^{\frac{3}{2}}} s^{k+2}\left\{\sum_{h=0}^{\left[\frac{1}{3}(k+2)\right]} \frac{1}{h !}\left(-\frac{1}{12}\right)^{h} \frac{1}{\Gamma(k+3-3 h)} s^{-3 h} \ln s\right. \\
& \left.+(-1)^{k+1} \sum_{h=\left[\frac{1}{3}(k+2)\right]+1}^{\infty} \frac{1}{h !}\left(\frac{1}{12}\right)^{h} \Gamma(3 h-k-2) s^{-3 h}\right\}
\end{aligned}
$$

in the double scaling limit (2.2), where $[x]$ denotes the greatest integer less than or equal to $x$. We can see that the infinite series in the bracket on the r.h.s. gives the genus expansion where the power of $g_{s}^{2} \propto s^{-3}$ counts the number of handles. The suffix "univ." on the 1.h.s. means that the most dominant nonanalytic term at $s=0$ is taken in the limit (2.2) (the universal part). The overall factor $N^{-\frac{2}{3}(k+2)}$ can be absorbed in the "wave function renormalization" of the operator $\frac{1}{N} \operatorname{tr} \phi^{2 k+1}$.

\subsection{Borel resummation}

The second line on the r.h.s. in (4.1) is a series exhibiting factorial growth as $\frac{\Gamma(3 h-k-2)}{h !} \sim$ $(2 h)$ !, which is a characteristic feature of string perturbation series and gives further support that the matrix model describes a string theory in the double scaling limit [98].

The factorial growth means that (4.1) is a divergent series with convergence radius zero. In order to try to make the series well-defined, let us apply the Borel resummation technique to (4.1). It amounts to inserting

$$
1=\frac{1}{\Gamma(2 h+1)} \int_{0}^{\infty} d z z^{2 h} e^{-z}
$$

into (4.1) and interchanging the order of the sum on $h$ and the integral on $z$. Use of Stirling's formula $\Gamma(x) \sim \sqrt{2 \pi} x^{x-\frac{1}{2}} e^{-x}\left[1+\frac{1}{12 x}+\mathcal{O}\left(x^{-2}\right)\right](x \rightarrow \infty)$ leads to

$$
\begin{aligned}
\frac{\Gamma(3 h-k-2)}{h ! \Gamma(2 h+1)} \sim & \frac{1}{2 \sqrt{\pi} 3^{k+\frac{5}{2}}}\left(\frac{27}{4}\right)^{h} h^{-k-\frac{7}{2}} \\
& \times\left[1+\left\{(k+2)(k+3)-\frac{7}{12}\right\} \frac{1}{6 h}+\mathcal{O}\left(h^{-2}\right)\right]
\end{aligned}
$$

for large $h$. In addition, a binomial coefficient $\left(\begin{array}{l}\alpha \\ h\end{array}\right)$ with $\alpha \notin \boldsymbol{Z}$ asymptotically behaves

$$
\begin{aligned}
\left(\begin{array}{l}
\alpha \\
h
\end{array}\right) & =(-1)^{h+1} \frac{\sin (\pi \alpha)}{\pi} \Gamma(\alpha+1) \frac{\Gamma(h-\alpha)}{h !} \\
& \sim(-1)^{h+1} \frac{\sin (\pi \alpha)}{\pi} \Gamma(\alpha+1) h^{-\alpha-1}\left[1+\frac{\alpha(\alpha+1)}{2 h}+\mathcal{O}\left(h^{-2}\right)\right] .
\end{aligned}
$$


Combining these two, we can express (4.3) as an expansion by binomial coefficients: ${ }^{4}$

$$
\begin{aligned}
\frac{\Gamma(3 h-k-2)}{h ! \Gamma(2 h+1)} \sim & \frac{(-1)^{h+k+1} \sqrt{\pi}}{2 \cdot 3^{k+\frac{5}{2}}}\left(\frac{27}{4}\right)^{h}\left[\frac{1}{\Gamma\left(k+\frac{7}{2}\right)}\left(\begin{array}{c}
k+\frac{5}{2} \\
h
\end{array}\right)\right. \\
+\frac{12 k^{2}+78 k+125}{36} & \left.\frac{1}{\Gamma\left(k+\frac{9}{2}\right)}\left(\begin{array}{c}
k+\frac{7}{2} \\
h
\end{array}\right)+\mathcal{O}\left(h^{-k-\frac{11}{2}}\right)\right]
\end{aligned}
$$

Then the Borel resummed series of (4.1) becomes

$$
\begin{aligned}
& \left.N^{\frac{2}{3}(k+2)}\left\langle\frac{1}{N} \operatorname{tr} \phi^{2 k+1}\right\rangle^{(1,0)}\right|_{\text {O-inst., univ. }} ^{\text {B.R. }} \\
& =\frac{1}{4 \pi} \frac{s^{k+2}}{3^{k+\frac{5}{2}}\left(k+\frac{3}{2}\right)\left(k+\frac{5}{2}\right)}\left[\int_{0}^{\infty} d z\left(1-\frac{z^{2}}{z_{0}^{2}}\right)^{k+\frac{5}{2}} e^{-z}\right. \\
& \left.\quad+\frac{12 k^{2}+78 k+125}{36\left(k+\frac{7}{2}\right)} \int_{0}^{\infty} d z\left(1-\frac{z^{2}}{z_{0}^{2}}\right)^{k+\frac{7}{2}} e^{-z}+\cdots\right]
\end{aligned}
$$

with

$$
z_{0} \equiv \frac{4}{3} s^{\frac{3}{2}}
$$

Here the ellipsis stands for integrals containing higher powers of $\left(1-\frac{z^{2}}{z_{0}^{2}}\right)$ and lower genus contributions up to $h=\left[\frac{1}{3}(k+2)\right]$. The former contributes to a series of ambiguities as well as the first two terms, whereas the latter is a finite sum providing nothing ambiguous. The integrals in (4.6) yield ambiguities due to the cut of the integrands $\left[z_{0},+\infty\right)$ on the integration contour. As depicted in figure 2, there are two ways to avoid the cut in the integrals of $z$, but the result will change depending on which choice we take. This means that the divergent series cannot be made well-defined (non-Borel summable) for the zeroinstanton sector alone. However, by taking account of contributions from nonzero instanton numbers, there is a possibility that ambiguity arising there cancels the ambiguity from the zero-instanton sector. Therefore, the theory is free from ambiguity and well-defined as a whole. This is the idea of resurgence. In the following, we will actually see that a series of ambiguities from (4.6) cancels that from one-instanton contribution (up to the next-to-leading order).

In order to identify the precise form of ambiguities, let us give a tiny imaginary part to $s: s \rightarrow s \pm i \epsilon$ with $\epsilon>0$. For $s+i \epsilon(s-i \epsilon)$, the integration contour of $z$ in (4.6) shifts slightly below (above) the positive real axis to $C_{+}\left(C_{-}\right)$as in figure 2 . Then the ambiguities are given as difference between contribution from $s+i \epsilon\left(C_{+}\right)$and that from

\footnotetext{
${ }^{4}$ For $m=0,1,2, \cdots,\left(\begin{array}{c}k+\frac{5}{2}+m \\ h\end{array}\right)$ is a quantity of $\mathcal{O}\left(h^{-k-\frac{7}{2}-m}\right)$ from eq. (4.4).
} 


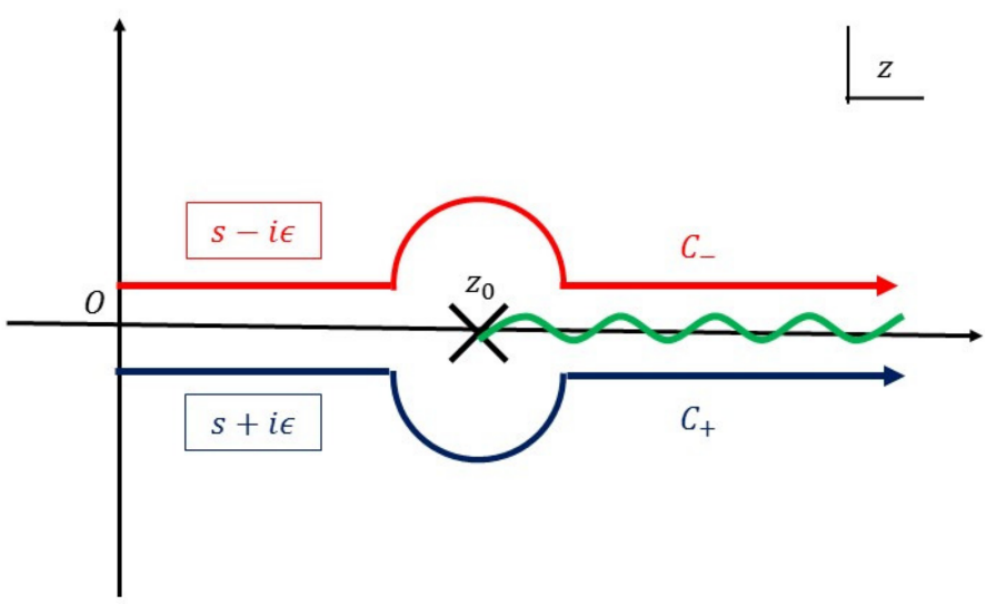

Figure 2. Integration contours $C_{+}$and $C_{-}$on the Borel plane.

$s-i \epsilon\left(C_{-}\right)$, i.e., integrated discontinuity of the integrands across the cut:

$$
\begin{aligned}
& \text { (Ambiguities of }(4.6))\left.\equiv(4.6)\right|_{s \rightarrow s+i \epsilon}-\left.(4.6)\right|_{s \rightarrow s-i \epsilon} \\
& =i(-1)^{k} \frac{1}{2 \pi} \frac{s^{k+2}}{3^{k+\frac{5}{2}}\left(k+\frac{3}{2}\right)\left(k+\frac{5}{2}\right)}\left[\int_{z_{0}}^{\infty} d z\left(\frac{z^{2}}{z_{0}^{2}}-1\right)^{k+\frac{5}{2}} e^{-z}\right. \\
& \left.\quad-\frac{12 k^{2}+78 k+125}{36\left(k+\frac{7}{2}\right)} \int_{z_{0}}^{\infty} d z\left(\frac{z^{2}}{z_{0}^{2}}-1\right)^{k+\frac{7}{2}} e^{-z}+\cdots\right] \\
& =i(-1)^{k} \frac{1}{3^{\frac{1}{2}} \pi^{\frac{3}{2}}} \frac{\Gamma\left(k+\frac{3}{2}\right)}{2^{k+2} s^{\frac{1}{2} k+1}}\left[K_{k+3}\left(z_{0}\right)-\frac{12 k^{2}+78 k+125}{18 z_{0}} K_{k+4}\left(z_{0}\right)+\cdots\right],
\end{aligned}
$$

where the ellipsis in the last line stands for terms of modified Bessel function $K_{k+3+\nu}\left(z_{0}\right)$ with $\nu \geq 2$. They are accompanied with $z_{0}^{-\nu}$ as in the second term and hence suppressed by $s^{-3 \nu / 2}$ compared to the first term. By using the asymptotic form

$$
K_{\nu}(z) \sim \sqrt{\frac{\pi}{2 z}} e^{-z}\left[1+\frac{4 \nu^{2}-1}{8 z}+\mathcal{O}\left(z^{-2}\right)\right] \quad(z \rightarrow+\infty)
$$

we finally find

$$
(\text { Ambiguities of }(4.6))=i(-1)^{k} \frac{\Gamma\left(k+\frac{3}{2}\right)}{2^{k+\frac{7}{2}} \pi} \frac{e^{-\frac{4}{3} s^{\frac{3}{2}}}}{s^{\frac{k}{2}+\frac{7}{4}}}\left[1-\frac{1}{8 s^{\frac{3}{2}}}\left(k^{2}+8 k+\frac{185}{12}\right)+\mathcal{O}\left(s^{-3}\right)\right] .
$$

Notice that the exponential factor $e^{-\frac{4}{3} s^{\frac{3}{2}}}$ is characteristic of the one-instanton contribution and its exponent comes from the value of the branch point $z_{0}$. This opens a profound connection between perturbative ambiguities and nonperturbative effects [99]. In addition, ambiguities from higher powers of $\left(\frac{z^{2}}{z_{0}^{2}}-1\right)$ seem to be related to higher corrections by holes and handles which are created by D-branes and closed strings, respectively. 


\section{Ambiguities in the one-instanton sector}

In the previous section, we have seen that the one-point functions of the odd-power operators provide divergent string perturbation series in the zero-instanton sector, and explicitly computed a series of ambiguities of its Borel resumed series at the leading and next-toleading orders. Here we find that another series of ambiguities appears in the one-instanton sector, and that these two series with different origins indeed cancel each other.

\subsection{One-point functions in the one-instanton sector}

By using the fact that the eigenvalue distribution of the standard Gaussian matrix model becomes the (diagonal) Airy kernel in the double scaling limit (2.2) [91, 100]:

$$
N^{\frac{1}{3}} \rho_{M}^{(\mathrm{G})}(x) \rightarrow K_{\mathrm{Ai}}(\xi, \xi) \equiv \mathrm{Ai}^{\prime}(\xi)^{2}-\xi \operatorname{Ai}(\xi)^{2}
$$

with $x=2+N^{-\frac{2}{3}} \xi$, we obtain the expression of (3.22) in the double scaling limit as

$$
\left.N^{\frac{2}{3}(k+2)}\left\langle\frac{1}{N} \operatorname{tr} \phi^{2 k+1}\right\rangle^{(1,0)}\right|_{0-\text { inst. }+1-\text { inst. }} \rightarrow \int_{-\infty}^{s} d \xi(s-\xi)^{k+\frac{1}{2}} K_{\mathrm{Ai}}(\xi, \xi) .
$$

From the relation of the Nicolai mapping $2+N^{-\frac{2}{3}} \xi=x=\mu^{2}-\lambda^{2}$, we see that the region $[a, b]$ for $\lambda$ in $(2.10)$ is mapped to $(-\infty, 0]$ for $\xi$, while the region $[0, a)$ is mapped to $(0, s]$ in the double scaling limit. ( $\xi=s$ corresponds to the location of the instanton $\lambda=0$.) Thus the latter gives contribution from the one-instanton sector:

$$
\left.N^{\frac{2}{3}(k+2)}\left\langle\frac{1}{N} \operatorname{tr} \phi^{2 k+1}\right\rangle^{(1,0)}\right|_{1-\text { inst. }} \rightarrow \int_{0}^{s} d \xi(s-\xi)^{k+\frac{1}{2}} K_{\mathrm{Ai}}(\xi, \xi) .
$$

\subsection{Saddle point method}

We consider contribution to the integral (5.3) from $\xi \sim s \gg 1$, which is expected to be comparable with the ambiguities (4.10). From asymptotic behavior of the Airy function, we find that the Airy kernel behaves as

$$
K_{\mathrm{Ai}}(\xi, \xi) \sim \frac{e^{-\frac{4}{3} \xi^{\frac{3}{2}}}}{8 \pi \xi}\left[1-\frac{17}{24 \xi^{\frac{3}{2}}}+\mathcal{O}\left(\xi^{-3}\right)\right] \quad(\xi \rightarrow \infty),
$$

where we take account of the expansion up to the next-to-leading order in order to compare the result in the zero-instanton sector (4.10). This leads us to consider the following integral

$$
\int_{0}^{s} d \xi(s-\xi)^{k+\frac{1}{2}} K_{\mathrm{Ai}}(\xi, \xi) \quad \rightarrow \quad \frac{1}{8 \pi} \int^{s} d \xi e^{-f(\xi)}
$$

with

$$
f(\xi) \equiv \frac{4}{3} \xi^{\frac{3}{2}}-\left(k+\frac{1}{2}\right) \ln (s-\xi)+\ln \xi+\frac{17}{24} \xi^{-\frac{3}{2}}+\mathcal{O}\left(\xi^{-3}\right) .
$$


For a while, we do not specify the lower bound of the integral region in (5.5), since our concern is contribution around $\xi=s$. Let us evaluate the integral (5.5) by a saddle point method. The saddle point around $\xi=s$ is found to be

$$
\xi_{*}=s+\frac{k+\frac{1}{2}}{2} s^{-\frac{1}{2}} v(s), \quad v(s) \equiv 1-\frac{k+\frac{5}{2}}{4} s^{-\frac{3}{2}}+\mathcal{O}\left(s^{-3}\right),
$$

where $v(s)$ represents corrections by subleading contributions. We see that the saddle point corresponding to the instanton $\xi_{*}=s$ slightly shifts due to the presence of the operator depending on $k$.

In the standard saddle point method, we first find a saddle point and rotate an original integration contour so that it will go along the steepest descent path passing through the saddle point. In our case, the original integration contour is not an infinite line, but a finite interval $[0, s]$, which is major difference from the standard case. The region $[0, s]$ is along the steepest descent path, but it terminates at the branch point $\xi=s$. The saddle point (5.7) is not in the interval $[0, s]$, but on the branch cut $[s, \infty)$. This can be treated by the shift $s \rightarrow s \pm i \epsilon$ as in section 4 . Here we rotate the contour by the angle $\pi$ (or $-\pi$ ) around $\xi=s$. The rotated contour goes in the opposite direction decreasing $\xi$ on the real axis, passes through $\xi_{*}$ and ends at $\xi=s$. We should choose the $\pi$ rotation or the $-\pi$ rotation of the contour of $\xi$ in accordance with $s \rightarrow s+i \epsilon$ or $s \rightarrow s-i \epsilon$, respectively.

Here it is worth noticing that what resurgence implies in the present setting. We first note that (3.22) itself is a well-defined real quantity without ambiguity, and we are interested in it after taking the double scaling limit. Because of technical difficulty, without explicit computation of the integral (3.22), we are trying to deduce its expression in the double scaling limit in the form of trans-series. Quantity in each instanton sector would have ambiguities, but resurgence ensures that reflecting the well-definedness of the original expression, such ambiguities are expected to cancel among instanton sectors. Even if the integration region does not contain a saddle point, we should develop perturbation theory around it in order to construct trans-series. As we will see later, information of the original contour can be included in how to rotate the contour and the end point of the rotated contour.

Let us go back to the computation. In calculating $f\left(\xi_{*}\right)$, we should use the shift $s \rightarrow s \pm i \epsilon$ to the term of $\ln \left(s-\xi_{*}\right)$ :

$$
\ln \left(s-\xi_{*}\right) \rightarrow \ln \left(s \pm i \epsilon-\xi_{*}\right)=\ln \left(\xi_{*}-s\right) \pm i \pi \quad(s \rightarrow s \pm i \epsilon)
$$

Then

$$
\begin{aligned}
f\left(\xi_{*}\right)= & \pm i \pi\left(k+\frac{1}{2}\right)+\tilde{f}\left(\xi_{*}\right), \\
\tilde{f}\left(\xi_{*}\right) \equiv & \frac{4}{3} s^{\frac{3}{2}}+\left(\frac{k}{2}+\frac{5}{4}\right) \ln s-\left(k+\frac{1}{2}\right) \ln \left(\frac{k+\frac{1}{2}}{2}\right)+k+\frac{1}{2} \\
& +\frac{1}{8}\left\{\left(k+\frac{1}{2}\right)\left(k+\frac{9}{2}\right)+\frac{17}{3}\right\} s^{-\frac{3}{2}}+\mathcal{O}\left(s^{-3}\right),
\end{aligned}
$$


while $\xi$-derivatives have no ambiguity:

$$
\begin{aligned}
f^{\prime \prime}\left(\xi_{*}\right) & =\frac{4 s}{k+\frac{1}{2}} \frac{1}{v(s)^{2}}+s^{-\frac{1}{2}}+\mathcal{O}\left(s^{-2}\right), \\
f^{(n)}\left(\xi_{*}\right) & =(-1)^{n}\left(k+\frac{1}{2}\right) \Gamma(n)\left(\frac{2 s^{\frac{1}{2}}}{k+\frac{1}{2}} \frac{1}{v(s)}\right)^{n}\left(1+\mathcal{O}\left(s^{-3}\right)\right) \quad(n \geq 3) .
\end{aligned}
$$

The Taylor expansion of $f(\xi)$ is summed up as

$$
\begin{aligned}
f(\xi) & =f\left(\xi_{*}\right)+\sum_{n=2}^{\infty} \frac{1}{n !} f^{(n)}\left(\xi_{*}\right) x^{n} \\
& =f\left(\xi_{*}\right)+\frac{2 s^{\frac{1}{2}} x}{v(s)}-\left(k+\frac{1}{2}\right) \ln \left(1+\frac{2 s^{\frac{1}{2}}}{k+\frac{1}{2}} \frac{x}{v(s)}\right)+\frac{1}{2} s^{-\frac{1}{2}} x^{2}+\mathcal{O}\left(s^{-3}\right)
\end{aligned}
$$

with $x=\xi-\xi_{*}$. Looking at the factor of the Gaussian integral $f^{\prime \prime}\left(\xi_{*}\right)$ in (5.11), we can regard $x$ as a quantity at most $\mathcal{O}\left(s^{-\frac{1}{2}}\right)$. From this, we find that all the terms in the Taylor expansion are of the same order and should be kept.

By changing the integration variable to

$$
t=\frac{2 s^{\frac{1}{2}}}{v(s)} x
$$

in order to zoom in the vicinity of the saddle point, the upper bound of the integral $x=s-\xi_{*}$ still remains a finite value $t=-k-\frac{1}{2}$, whereas the lower bound becomes far away from the saddle point by $\mathcal{O}\left(s^{\frac{1}{2}}\right)$. The integral we should evaluate becomes ${ }^{5}$

$$
\begin{array}{rl}
\frac{1}{8 \pi} \int^{s} & d \xi e^{-f(\xi)} \\
\quad= \pm \frac{i}{8 \pi}(-1)^{k} e^{-\tilde{f}\left(\xi_{*}\right)} \frac{v(s)}{2 s^{\frac{1}{2}}} \int_{\infty}^{-k-\frac{1}{2}} d t\left(1+\frac{t}{k+\frac{1}{2}}\right)^{k+\frac{1}{2}} e^{-t}\left[1-\frac{v(s)^{2}}{8 s^{\frac{3}{2}}} t^{2}+\mathcal{O}\left(s^{-3}\right)\right] \\
\quad=\mp i(-1)^{k} \frac{\Gamma\left(k+\frac{3}{2}\right)}{2^{k+\frac{9}{2}} \pi} \frac{e^{-\frac{4}{3} s^{\frac{3}{2}}}}{s^{\frac{k}{2}+\frac{7}{4}}}\left[1-\frac{1}{8}\left(k^{2}+8 k+\frac{185}{12}\right) s^{-\frac{3}{2}}+\mathcal{O}\left(s^{-2}\right)\right]
\end{array}
$$

We end up with

$$
\begin{aligned}
& \left.(\text { Ambiguities of }(5.3)) \equiv(5.14)\right|_{s \rightarrow s+i \epsilon}-\left.(5.14)\right|_{s \rightarrow s-i \epsilon} \\
& \qquad=-i(-1)^{k} \frac{\Gamma\left(k+\frac{3}{2}\right)}{2^{k+\frac{7}{2}} \pi} \frac{e^{-\frac{4}{3} s^{\frac{3}{2}}}}{s^{\frac{k}{2}+\frac{7}{4}}}\left[1-\frac{1}{8}\left(k^{2}+8 k+\frac{185}{12}\right) s^{-\frac{3}{2}}+\mathcal{O}\left(s^{-3}\right)\right],
\end{aligned}
$$

\footnotetext{
${ }^{5}$ We can check that the integrand does not depend on the contour rotation by $\pi$ or $-\pi$. Setting $s-\xi=$ $r e^{i \theta}, \theta$ is supposed to rotate from 0 to $\pm \pi$ in accordance with $s \rightarrow s \pm i \epsilon$ as mentioned before. Then $t$ becomes $t=-k-\frac{1}{2}-\frac{2 s^{\frac{1}{2}}}{v(s)} r e^{i \theta}$. Only the subtle factor in the integrand $\left(1+\frac{t}{k+\frac{1}{2}}\right)^{k+\frac{1}{2}}$ is written as $\left(e^{\mp i \pi} \frac{2 s^{\frac{1}{2}}}{\left(k+\frac{1}{2}\right) v(s)} r e^{i \theta}\right)^{k+\frac{1}{2}}$ due to $-s \mp i \epsilon=e^{\mp i \pi} s$. Hence it becomes the same irrespective of rotating $\theta$ by
} 
which precisely cancels the series of ambiguities in the zero-instanton sector (4.10) regarding the leading and next-to-leading contributions. In the above derivation, we identify the origin of ambiguities as the saddle point value of the integrand, more precisely, of the operator $\frac{1}{N} \operatorname{tr} \phi^{2 k+1}$. In fact, the imaginary ambiguities come from the logarithmic term (5.8) whose origin is the operator $(s-\xi)^{k+\frac{1}{2}}$ after the Nicolai mapping. It is reasonable because the partition function or even-power operators $\frac{1}{N} \operatorname{tr} \phi^{2 k}$ do not have any ambiguity and hence the existence of ambiguities must not depend on the eigenvalue distribution or the Airy kernel, but on the kind of operators.

In (5.12), we manage to sum up the Taylor series to obtain the logarithmic term which is a key to quickly derive the Gamma function $\Gamma\left(k+\frac{3}{2}\right)$ in the ambiguities (5.14). If we perform the ordinary saddle point calculation, i.e., Gaussian integral over the whole real axis by bringing down the higher terms of $n \geq 3$ in (5.12) from the exponent, the Gamma function will appear in the form of the asymptotic series as $k+\frac{1}{2}$ grows. ${ }^{6}$ A similar situation was found in [27] where difference between a Gamma function factor and its form of Stirling's formula is supplemented by terms whose order is higher than quadratic. There, it is necessary to go beyond the one-loop determinant since quasi zero-modes appear. In our case, there is no such zero-mode since $f^{\prime \prime}\left(\xi_{*}\right)$ is positive definite, but still all order terms needs to be taken into account in order to confirm resurgence. It would be interesting that in both cases we need all order terms in the saddle point method for different reasons.

In order to derive the trans-series around the instanton saddle for the finite interval, we took a prescription to rotate the integration contour so that it will pass the saddle point with taking care of the direction and the end point of the original contour. We have explicitly seen that this prescription realizes the cancellation not only at the leading order but also at the next-to-leading order. This result supports validity of our prescription. Some powerful technique will be necessary to check the cancellation to all orders or at the level of higher instanton numbers. It is also desirable to give more justification of this prescription from the viewpoint of general theory on resurgence applied to an interval.

Finally, concerning our motivation mentioned in Introduction, we make a comment on a relation to physics, in particular spontaneous supersymmetry breaking. As shown in [90] (eqs. (5.25) and (5.26) there $^{7}$ ), its order parameter is given by the Airy kernel (5.1) as

$$
N^{\frac{4}{3}}\left\langle\frac{1}{N} \operatorname{tr}\left(\phi^{2}-\mu^{2}\right)\right\rangle^{(1,0)}=K_{\mathrm{Ai}}(s, s)+\cdots,
$$

where the ellipsis stands for contribution from higher instantons. As explicitly seen from (5.4), this expression can be interpreted as contribution of the instanton, namely an isolated eigenvalue at the top of the potential. It would be important to understand physical aspects of a connection between the ambiguity computed here and the order parameter (5.16).

\footnotetext{
${ }^{6}$ From $(5.11), x=\xi-\xi_{*}$ can be regarded as a quantity at most $\mathcal{O}\left(\left(k+\frac{1}{2}\right)^{\frac{1}{2}}\right)$ in the Gaussian integral with respect to the $k$-dependence. Thus the $n$-th order term in $(5.12)$ is suppressed as $\mathcal{O}\left(\left(k+\frac{1}{2}\right)^{1-\frac{n}{2}}\right)$.

${ }^{7}$ The variable $t$ there should be read as $s / 4$.
} 


\section{Conclusions and discussions}

In this paper, we have investigated resurgence structure in the one-point functions of nonsupersymmetric operators in the supersymmetric double-well matrix model which is proposed as nonperturbative formulation of two-dimensional type IIA superstring theory. In the zero-instanton sector, the Borel resummation technique is applied to a divergent string perturbation series, and a series of ambiguities arises depending on how to avoid the cut on the Borel plane. In the one-instanton sector, a special care is necessary for the integration contour of a finite interval which does not pass through a saddle point. Another series of ambiguities arise from the integrand itself evaluated at the saddle point. We have confirmed that these two series of ambiguities cancel each other both at the leading and next-to-leading order. Our prescription of the integration contour in the one-instanton sector is worth studying further and needs to be understood from the viewpoint of resurgence theory extended to cases of integrals over finite intervals.

Another interesting question is how resurgence structure changes for other correlation functions in the same model. In fact, we have developed a derivation of multi-point functions of odd-power operators in [96] by using the result in the Gaussian matrix model [97]. There, it should be possible to read off large-order behavior of genus expansion for the twopoint (or multi-point) functions of the odd-power operators [109]. It would be interesting to check that it again shows the stringy growth of the expansion coefficient as $(2 h)$ ! and to find structure of singularities on the Borel plane. On the other hand, in the one-instanton sector, we need to treat off-diagonal components of the Airy kernel $K_{\mathrm{Ai}}(\xi, \eta)$, and it is expected that we find richer resurgence structure with variety of saddle points and steepest descent paths (Lefschetz thimbles).

It is also anticipated that our analysis here can be extended to other models where the Nicolai mapping is available. If they are mapped to the Gaussian matrix model via the Nicolai mapping, correlation functions will be calculated from the result in the Gaussian matrix model as in (3.7). If one is interested in the soft edge scaling limit, studies on the Airy kernel and resurgence structure in this paper will be useful there as well.

\section{Acknowledgments}

We would like to thank Toshiaki Fujimori, Tatsuhiro Misumi, Shinsuke Nishigaki, Norisuke Sakai, Yuya Tanizaki, Mithat Ünsal and Sen Zhang for useful discussions and comments. The work of T. K. is supported in part by a Grant-in-Aid for Scientific Research (C), 16K05335. The work of F. S. is supported in part by a Grant-in-Aid for Scientific Research (C), 25400289. We would like to thank the Yukawa Institute for Theoretical Physics at Kyoto University for hospitality during the workshop YITP-W-17-08 "Strings and Fields 2017," and RIKEN during the workshop "RIMS-iTHEMS International Workshop on Resurgence Theory," where part of this work was carried out.

Open Access. This article is distributed under the terms of the Creative Commons Attribution License (CC-BY 4.0), which permits any use, distribution and reproduction in any medium, provided the original author(s) and source are credited. 


\section{References}

[1] J. Ecalle, Les fonctions resurgentes. I-III, Publ. Math. Orsay, France (1981).

[2] F. Pham, Vanishing homologies and the $n$ variable saddle point method, Proc. Symp. Pure Math 2 (1983) 319.

[3] M.V. Berry and C.J. Howls, Hyperasymptotics for integrals with saddles, Proc. R. Soc. Longon A 434 (1991) 657.

[4] C.J. Howls, Hyperasymptotics for multidimensional integrals, exact remainder terms and the global connection problem, Proc. R. Soc. London 453 (1997) 2271.

[5] E. Delabaere and C.J. Howls, Global asymptotics for multiple integrals with boundaries, Duke Math. J. 112 (2002) 199.

[6] O. Costin, Asymptotics and Borel summability, Chapman Hall, U.S.A. (2008).

[7] D. Sauzin, Resurgent functions and splitting problems, RIMS Kokyuroku 1493 (2006), arXiv:0706.0137.

[8] D. Sauzin, Introduction to 1-summability and resurgence, arXiv:1405.0356.

[9] G. Alvarez and C. Casares, Exponentially small corrections in the asymptotic expansion of the eigenvalues of the cubic anharmonic oscillator, J. Phys. A 33 (2000) 5171.

[10] G. Alvarez and C. Casares, Uniform asymptotic and JWKB expansions for anharmonic oscillators, J. Phys. A 33 (2000) 2499.

[11] G. Alvarez, Langer-Cherry derivation of the multi-instanton expansion for the symmetric double well, J. Math. Phys. 45 (2004) 3095.

[12] J. Zinn-Justin and U.D. Jentschura, Multi-instantons and exact results I: conjectures, WKB expansions and instanton interactions, Annals Phys. 313 (2004) 197 [quant-ph/0501136] [INSPIRE].

[13] J. Zinn-Justin and U.D. Jentschura, Multi-instantons and exact results II: specific cases, higher-order effects and numerical calculations, Annals Phys. 313 (2004) 269 [quant-ph/0501137] [INSPIRE].

[14] U.D. Jentschura, A. Surzhykov and J. Zinn-Justin, Multi-instantons and exact results. III: unification of even and odd anharmonic oscillators, Annals Phys. 325 (2010) 1135 [INSPIRE].

[15] U.D. Jentschura and J. Zinn-Justin, Multi-instantons and exact results. IV: Path integral formalism, Annals Phys. 326 (2011) 2186 [INSPIRE].

[16] G.V. Dunne and M. Ünsal, Generating nonperturbative physics from perturbation theory, Phys. Rev. D 89 (2014) 041701 [arXiv:1306.4405] [INSPIRE].

[17] G.V. Dunne and M. Ünsal, Uniform WKB, multi-instantons and resurgent trans-series, Phys. Rev. D 89 (2014) 105009 [arXiv:1401.5202] [INSPIRE].

[18] G.V. Dunne and M. Ünsal, WKB and resurgence in the Mathieu equation, arXiv: 1603.04924 [INSPIRE].

[19] G. Basar, G.V. Dunne and M. Ünsal, Resurgence theory, ghost-instantons and analytic continuation of path integrals, JHEP 10 (2013) 041 [arXiv:1308.1108] [INSPIRE]. 
[20] M.A. Escobar-Ruiz, E. Shuryak and A.V. Turbiner, Three-loop correction to the instanton density. I. The quartic double well potential, Phys. Rev. D 92 (2015) 025046 [Erratum ibid. D 92 (2015) 089902] [arXiv: 1501.03993] [INSPIRE].

[21] M.A. Escobar-Ruiz, E. Shuryak and A.V. Turbiner, Three-loop correction to the instanton density. II. The sine-Gordon potential, Phys. Rev. D 92 (2015) 025047 [arXiv:1505. 05115] [INSPIRE].

[22] T. Misumi, M. Nitta and N. Sakai, Resurgence in sine-Gordon quantum mechanics: exact agreement between multi-instantons and uniform WKB, JHEP 09 (2015) 157 [arXiv: 1507.00408] [INSPIRE].

[23] A. Behtash et al., Complexified path integrals, exact saddles and supersymmetry, Phys. Rev. Lett. 116 (2016) 011601 [arXiv:1510.00978] [INSPIRE].

[24] A. Behtash et al., Toward Picard-Lefschetz theory of path integrals, complex saddles and resurgence, arXiv:1510.03435 [INSPIRE].

[25] A. Behtash et al., Critical points at infinity, non-Gaussian saddles and bions, JHEP 06 (2018) 068 [arXiv: 1803.11533] [INSPIRE].

[26] I. Gahramanov and K. Tezgin, Remark on the Dunne-Ünsal relation in exact semiclassics, Phys. Rev. D 93 (2016) 065037 [arXiv:1512.08466] [InSPIRE].

[27] T. Fujimori et al., Nonperturbative contributions from complexified solutions in $\mathbb{C} P^{N-1}$ models, Phys. Rev. D 94 (2016) 105002 [arXiv: 1607.04205] [INSPIRE].

[28] T. Fujimori et al., Exact resurgent trans-series and multibion contributions to all orders, Phys. Rev. D 95 (2017) 105001 [arXiv: 1702.00589] [INSPIRE].

[29] T. Fujimori et al., Resurgence structure to all orders of multi-bions in deformed SUSY quantum mechanics, PTEP 2017 (2017) 083B02 [arXiv:1705.10483] [INSPIRE].

[30] G.V. Dunne and M. Ünsal, Deconstructing zero: resurgence, supersymmetry and complex saddles, JHEP 12 (2016) 002 [arXiv: 1609.05770] [INSPIRE].

[31] C. Kozçaz, T. Sulejmanpasic, Y. Tanizaki and M. Ünsal, Cheshire cat resurgence, self-resurgence and quasi-exact solvable systems, Commun. Math. Phys. 364 (2018) 835 [arXiv: 1609.06198] [INSPIRE].

[32] M. Serone, G. Spada and G. Villadoro, Instantons from perturbation theory, Phys. Rev. D 96 (2017) 021701 [arXiv: 1612.04376] [INSPIRE].

[33] M. Serone, G. Spada and G. Villadoro, The power of perturbation theory, JHEP 05 (2017) 056 [arXiv: 1702.04148 ] [INSPIRE].

[34] G. Basar, G.V. Dunne and M. Ünsal, Quantum geometry of resurgent perturbative/nonperturbative relations, JHEP 05 (2017) 087 [arXiv:1701.06572] [INSPIRE].

[35] G. Álvarez and H.J. Silverstone, A new method to sum divergent power series: educated match, J. Phys. Comm. 1 (2017) 025005 [arXiv: 1706.00329] [InSPIRE].

[36] M. Mariño, R. Schiappa and M. Weiss, Multi-instantons and multi-cuts, J. Math. Phys. 50 (2009) 052301 [arXiv:0809.2619] [INSPIRE].

[37] S. Garoufalidis, A. Its, A. Kapaev and M. Mariño, Asymptotics of the instantons of Painlevé I, Int. Math. Res. Not. 2012 (2012) 561 [arXiv:1002.3634] [INSPIRE]. 
[38] C.-T. Chan, H. Irie and C.-H. Yeh, Stokes phenomena and non-perturbative completion in the multi-cut two-matrix models, Nucl. Phys. B 854 (2012) 67 [arXiv:1011.5745] [INSPIRE].

[39] C.-T. Chan, H. Irie and C.-H. Yeh, Stokes phenomena and quantum integrability in non-critical string/M theory, Nucl. Phys. B 855 (2012) 46 [arXiv:1109.2598] [INSPIRE].

[40] R. Schiappa and R. Vaz, The resurgence of instantons: multi-cut Stokes phases and the Painlevé II equation, Commun. Math. Phys. 330 (2014) 655 [arXiv:1302.5138] [InSPIRE].

[41] M. Mariño, Open string amplitudes and large order behavior in topological string theory, JHEP 03 (2008) 060 [hep-th/0612127] [inSPIRE].

[42] M. Mariño, Nonperturbative effects and nonperturbative definitions in matrix models and topological strings, JHEP 12 (2008) 114 [arXiv:0805.3033] [INSPIRE].

[43] M. Mariño, R. Schiappa and M. Weiss, Nonperturbative effects and the large-order behavior of matrix models and topological strings, Commun. Num. Theor. Phys. 2 (2008) 349 [arXiv:0711.1954] [INSPIRE].

[44] S. Pasquetti and R. Schiappa, Borel and Stokes nonperturbative phenomena in topological string theory and $c=1$ matrix models, Annales Henri Poincaré 11 (2010) 351 [arXiv: 0907 .4082] [INSPIRE].

[45] I. Aniceto, R. Schiappa and M. Vonk, The resurgence of instantons in string theory, Commun. Num. Theor. Phys. 6 (2012) 339 [arXiv:1106.5922] [INSPIRE].

[46] I. Aniceto and R. Schiappa, Nonperturbative ambiguities and the reality of resurgent transseries, Commun. Math. Phys. 335 (2015) 183 [arXiv:1308.1115] [InSPIRE].

[47] I. Aniceto and R. Schiappa, Nonperturbative ambiguities and the reality of resurgent transseries, Commun. Math. Phys. 335 (2015) 183 [arXiv:1308.1115] [InSPIRE].

[48] R. Couso-Santamaría, J.D. Edelstein, R. Schiappa and M. Vonk, Resurgent transseries and the holomorphic anomaly: nonperturbative closed strings in local $\mathbb{C P}^{2}$, Commun. Math. Phys. 338 (2015) 285 [arXiv:1407.4821] [INSPIRE].

[49] M. Vonk, Resurgence and topological strings, Proc. Symp. Pure Math. 93 (2015) 221 [arXiv: 1502.05711] [INSPIRE].

[50] R. Couso-Santamaría, R. Schiappa and R. Vaz, On asymptotics and resurgent structures of enumerative Gromov-Witten invariants, Commun. Num. Theor. Phys. 11 (2017) 707 [arXiv: 1605.07473] [INSPIRE].

[51] R. Couso-Santamaría, M. Mariño and R. Schiappa, Resurgence matches quantization, J. Phys. A 50 (2017) 145402 [arXiv:1610.06782] [InSPIRE].

[52] A. Grassi, M. Mariño and S. Zakany, Resumming the string perturbation series, JHEP 05 (2015) 038 [arXiv: 1405.4214] [INSPIRE].

[53] M. Mariño, Lectures on non-perturbative effects in large $N$ gauge theories, matrix models and strings, Fortsch. Phys. 62 (2014) 455 [arXiv:1206.6272] [INSPIRE].

[54] D. Dorigoni, An introduction to resurgence, trans-series and alien calculus, arXiv: 1411.3585 [INSPIRE].

[55] G.V. Dunne and M. Ünsal, What is QFT? Resurgent trans-series, Lefschetz thimbles and new exact saddles, PoS (LATTICE2015) 010 [arXiv:1511.05977] [INSPIRE]. 
[56] G.V. Dunne and M. Ünsal, New nonperturbative methods in quantum field theory: from large-N orbifold equivalence to bions and resurgence, Ann. Rev. Nucl. Part. Sci. 66 (2016) 245 [arXiv: 1601.03414$]$ [INSPIRE].

[57] I. Aniceto, G. Basar and R. Schiappa, A primer on resurgent transseries and their asymptotics, arXiv:1802.10441 [INSPIRE].

[58] I. Aniceto, The resurgence of the cusp anomalous dimension, J. Phys. A 49 (2016) 065403 [arXiv: 1506.03388] [INSPIRE].

[59] D. Dorigoni and Y. Hatsuda, Resurgence of the cusp anomalous dimension, JHEP 09 (2015) 138 [arXiv: 1506.03763] [INSPIRE].

[60] G. Arutyunov, D. Dorigoni and S. Savin, Resurgence of the dressing phase for $A d S_{5} \times S^{5}$, JHEP 01 (2017) 055 [arXiv: 1608.03797] [INSPIRE].

[61] G.V. Dunne and M. Ünsal, Resurgence and Trans-series in Quantum Field Theory: The $C P(N-1)$ Model, JHEP 11 (2012) 170 [arXiv:1210.2423] [inSPIRE].

[62] G.V. Dunne and M. Ünsal, Continuity and resurgence: towards a continuum definition of the $\mathbb{C P}^{(N-1)}$ model, Phys. Rev. D 87 (2013) 025015 [arXiv:1210.3646] [INSPIRE].

[63] A. Cherman, D. Dorigoni, G.V. Dunne and M. Ünsal, Resurgence in quantum field theory: nonperturbative effects in the principal chiral model, Phys. Rev. Lett. 112 (2014) 021601 [arXiv: 1308.0127] [INSPIRE].

[64] A. Cherman, D. Dorigoni and M. Ünsal, Decoding perturbation theory using resurgence: Stokes phenomena, new saddle points and Lefschetz thimbles, JHEP 10 (2015) 056 [arXiv: 1403.1277] [INSPIRE].

[65] T. Misumi, M. Nitta and N. Sakai, Neutral bions in the $\mathbb{C} P^{N-1}$ model, JHEP 06 (2014) 164 [arXiv: 1404.7225] [INSPIRE].

[66] T. Misumi, M. Nitta and N. Sakai, Classifying bions in Grassmann $\sigma$-models and non-Abelian gauge theories by D-branes, PTEP 2015 (2015) 033B02 [arXiv:1409.3444] [INSPIRE].

[67] T. Misumi, M. Nitta and N. Sakai, Neutral bions in the $\mathbb{C P}^{N-1}$ model for resurgence, J. Phys. Conf. Ser. 597 (2015) 012060 [arXiv:1412.0861] [InSPIRE].

[68] T. Misumi, M. Nitta and N. Sakai, Non-BPS exact solutions and their relation to bions in $\mathbb{C} P^{N-1}$ models, JHEP 05 (2016) 057 [arXiv: 1604.00839] [INSPIRE].

[69] M. Nitta, Fractional instantons and bions in the $O(N)$ model with twisted boundary conditions, JHEP 03 (2015) 108 [arXiv:1412.7681] [INSPIRE].

[70] M. Nitta, Fractional instantons and bions in the principal chiral model on $\mathbb{R}^{2} \times S^{1}$ with twisted boundary conditions, JHEP 08 (2015) 063 [arXiv:1503.06336] [INSPIRE].

[71] A. Behtash, T. Sulejmanpasic, T. Schäfer and M. Ünsal, Hidden topological angles and Lefschetz thimbles, Phys. Rev. Lett. 115 (2015) 041601 [arXiv:1502.06624] [INSPIRE].

[72] G.V. Dunne and M. Ünsal, Resurgence and dynamics of $O(N)$ and Grassmannian $\sigma$-models, JHEP 09 (2015) 199 [arXiv: 1505.07803] [INSPIRE].

[73] P.V. Buividovich, G.V. Dunne and S.N. Valgushev, Complex path integrals and saddles in two-dimensional gauge theory, Phys. Rev. Lett. 116 (2016) 132001 [arXiv:1512.09021] [INSPIRE]. 
[74] S. Demulder, D. Dorigoni and D.C. Thompson, Resurgence in $\eta$-deformed principal chiral models, JHEP 07 (2016) 088 [arXiv:1604.07851] [INSPIRE].

[75] T. Sulejmanpasic, Global symmetries, volume independence and continuity in quantum field theories, Phys. Rev. Lett. 118 (2017) 011601 [arXiv:1610.04009] [INSPIRE].

[76] S. Gukov, M. Mariño and P. Putrov, Resurgence in complex Chern-Simons theory, arXiv: 1605.07615 [INSPIRE].

[77] D. Gang and Y. Hatsuda, S-duality resurgence in SL(2) Chern-Simons theory, JHEP 07 (2018) 053 [arXiv: 1710.09994] [InSPIRE].

[78] P. Argyres and M. Ünsal, A semiclassical realization of infrared renormalons, Phys. Rev. Lett. 109 (2012) 121601 [arXiv:1204.1661] [INSPIRE].

[79] P.C. Argyres and M. Ünsal, The semi-classical expansion and resurgence in gauge theories: new perturbative, instanton, bion and renormalon effects, JHEP 08 (2012) 063 [arXiv:1206.1890] [INSPIRE].

[80] G.V. Dunne, M. Shifman and M. Ünsal, Infrared renormalons versus operator product expansions in supersymmetric and related gauge theories, Phys. Rev. Lett. 114 (2015) 191601 [arXiv: 1502 .06680] [INSPIRE].

[81] M. Yamazaki and K. Yonekura, From $4 d$ Yang-Mills to $2 d \mathbb{C P}^{N-1}$ model: IR problem and confinement at weak coupling, JHEP 07 (2017) 088 [arXiv: 1704.05852] [INSPIRE].

[82] J.G. Russo, A note on perturbation series in supersymmetric gauge theories, JHEP 06 (2012) 038 [arXiv: 1203.5061] [INSPIRE].

[83] I. Aniceto, J.G. Russo and R. Schiappa, Resurgent analysis of localizable observables in supersymmetric gauge theories, JHEP 03 (2015) 172 [arXiv:1410.5834] [INSPIRE].

[84] M. Honda, Borel summability of perturbative series in $4 D N=2$ and $5 D N=1$ supersymmetric theories, Phys. Rev. Lett. 116 (2016) 211601 [arXiv:1603.06207] [INSPIRE].

[85] M. Honda, How to resum perturbative series in $3 d N=2$ Chern-Simons matter theories, Phys. Rev. D 94 (2016) 025039 [arXiv: 1604.08653] [InSPIRE].

[86] M. Honda, Supersymmetric solutions and Borel singularities for $N=2$ supersymmetric Chern-Simons theories, Phys. Rev. Lett. 121 (2018) 021601 [arXiv:1710.05010] [inSPIRE].

[87] D. Dorigoni and P. Glass, The grin of Cheshire cat resurgence from supersymmetric localization, SciPost Phys. 4 (2018) 012 [arXiv:1711.04802] [INSPIRE].

[88] M. Honda and D. Yokoyama, Resumming perturbative series in the presence of monopole bubbling effects, arXiv:1711.10799 [INSPIRE].

[89] T. Fujimori, M. Honda, S. Kamata, T. Misumi and N. Sakai, Resurgence and Lefschetz thimble in three-dimensional $\mathcal{N}=2$ supersymmetric Chern-Simons matter theories, PTEP 2018 (2018) 123B03 [arXiv: 1805.12137] [INSPIRE].

[90] M.G. Endres, T. Kuroki, F. Sugino and H. Suzuki, SUSY breaking by nonperturbative dynamics in a matrix model for $2 D$ type IIA superstrings, Nucl. Phys. B 876 (2013) 758 [arXiv: 1308.3306] [INSPIRE].

[91] S.M. Nishigaki and F. Sugino, Tracy-Widom distribution as instanton sum of $2 D$ IIA superstrings, JHEP 09 (2014) 104 [arXiv:1405.1633] [INSPIRE]. 
[92] T. Kuroki and F. Sugino, Supersymmetric double-well matrix model as two-dimensional type IIA superstring on RR background, JHEP 03 (2014) 006 [arXiv:1306.3561] [INSPIRE].

[93] T. Kuroki and F. Sugino, New critical behavior in a supersymmetric double-well matrix model, Nucl. Phys. B 867 (2013) 448 [arXiv:1208.3263] [InSPIRE].

[94] T. Kuroki and F. Sugino, Spontaneous supersymmetry breaking in large- $N$ matrix models with slowly varying potential, Nucl. Phys. B 830 (2010) 434 [arXiv: 0909.3952] [InSPIRE].

[95] T. Kuroki and F. Sugino, Spontaneous supersymmetry breaking in matrix models from the viewpoints of localization and Nicolai mapping, Nucl. Phys. B 844 (2011) 409 [arXiv: 1009.6097] [INSPIRE].

[96] T. Kuroki and F. Sugino, One-point functions of non-SUSY operators at arbitrary genus in a matrix model for type IIA superstrings, Nucl. Phys. B 919 (2017) 325 [arXiv: 1609.01628] [INSPIRE].

[97] U. Haagerup and S. Thorbjørnsen, Asymptotic expansions for the Gaussian unitary ensemble, Infin. Dimens. Anal. Quantum Probab. Relat. Top. 15 (2012) 1250003 [arXiv:1004.3479].

[98] S.H. Shenker, The strength of nonperturbative effects in string theory, in The large $N$ expansion in quantum field theory and statistical physics, E. Brezin and S.R. Wadia, World Scientific, Singapore (1993).

[99] J. Zinn-Justin, Quantum field theory and critical phenomena, Oxford University Press, Oxford, U.K. (1996).

[100] C.A. Tracy and H. Widom, Level spacing distributions and the Airy kernel, Commun. Math. Phys. 159 (1994) 151 [hep-th/9211141] [INSPIRE].

[101] F. David, Nonperturbative effects in matrix models and vacua of two-dimensional gravity, Phys. Lett. B 302 (1993) 403 [hep-th/9212106] [INSPIRE].

[102] V.A. Kazakov and I.K. Kostov, Instantons in noncritical strings from the two matrix model, hep-th/0403152 [INSPIRE].

[103] M. Hanada et al., Loops versus matrices: The Nonperturbative aspects of noncritical string, Prog. Theor. Phys. 112 (2004) 131 [hep-th/0405076] [INSPIRE].

[104] H. Kawai, T. Kuroki and Y. Matsuo, Universality of nonperturbative effect in type 0 string theory, Nucl. Phys. B 711 (2005) 253 [hep-th/0412004] [INSPIRE].

[105] A. Sato and A. Tsuchiya, ZZ brane amplitudes from matrix models, JHEP 02 (2005) 032 [hep-th/0412201] [INSPIRE].

[106] N. Ishibashi and A. Yamaguchi, On the chemical potential of D-instantons in $c=0$ noncritical string theory, JHEP 06 (2005) 082 [hep-th/0503199] [INSPIRE].

[107] N. Ishibashi, T. Kuroki and A. Yamaguchi, Universality of nonperturbative effects in $c<1$ noncritical string theory, JHEP 09 (2005) 043 [hep-th/0507263] [INSPIRE].

[108] T. Kuroki and F. Sugino, T duality of the Zamolodchikov-Zamolodchikov brane, Phys. Rev. D 75 (2007) 044008 [hep-th/0612042] [INSPIRE].

[109] T. Kuroki and F. Sugino, Higher-genus amplitudes in SUSY double-well matrix model (II): Two-point functions to all orders, in preparation. 